\title{
Homogenization of a periodic semilinear elliptic degenerate PDE
}

\author{
Étienne Pardoux ${ }^{(a)} \quad$ Ahmadou Bamba Sow ${ }^{(b)}$
}

October 18, 2018

\begin{abstract}
(a) CMI, LATP-UMR 6632, Université de Provence, 39 rue F. Joliot Curie, Marseille cedex 13, FRANCE. email : pardoux@cmi.univ-mrs.fr

(b) LERSTAD, UFR S.A.T, Université Gaston Berger, BP 234, Saint-Louis, SENEGAL.

email : ahmadou-bamba.sow@ugb.edu.sn
\end{abstract}

\begin{abstract}
In this paper a semilinear elliptic PDE with rapidly oscillating coefficients is homogenized. The novetly of our result lies in the fact that we allow the second order part of the differential operator to be degenerate in some portion of $\mathbb{R}^{d}$.

Our fully probabilistic method is based on the connection between PDEs and BSDEs with random terminal time and the weak convergence of a class of diffusion processes.
\end{abstract}

Keywords : Poisson equation, ergodic theorem, backward stochastic differential equation with random time.

\section{Introduction}

The theory of homogenization tries to understand what equations should be used at a macroscopic level, in order to approximate the behavior of physical phenomena described at a microscopic level by equations with highly oscillatory coefficients. This theory has motivated the development of various notions of weak convergence in analysis, see in particular Tartar [18]. One way to understand such convergence, at least in the case of linear or certain semilinear equations with periodic coefficients is based on a probabilistic interpretation of the equation, see among others Freidlin [6], [7] in the linear case, Briand and Hu [3], Pardoux [12] and Delarue [4] in the semilinear case. The last three papers exploit the connection between BSDEs and semilinear PDEs, see Pardoux and Rascanu [15].

Recently Hairer and Pardoux [8] have generalized the probabilistic approach to the homogenization of linear second-order periodic PDEs with periodic coefficients to systems where the matrix of second order coefficients can be allowed to degenerate or even vanish 
on an open subset of $\mathbb{R}^{d}$. Those results have been extended to semilinear parabolic PDEs in Sow, Rhodes and Pardoux [17]. The aim of the present paper is to treat a class of semilinear elliptic PDEs, whose matrix of second order ocefficients is allowed to vanish in part of $\mathbb{R}^{d}$.

More precisely, we will study the homogenization of the elliptic Dirichlet boundary value problem in the smooth bounded domain $G \subset \mathbb{R}^{d}$ :

$$
\left\{\begin{aligned}
L_{\varepsilon} u^{\varepsilon}(x) & +f\left(\frac{x}{\varepsilon}, x, u^{\varepsilon}(x), \partial_{x} u^{\varepsilon}(x) \sigma\left(\frac{x}{\varepsilon}\right)\right)=0, \quad x \in G, \\
u^{\varepsilon}(x) & =g(x), \quad x \in \partial G
\end{aligned}\right.
$$

where the second order differential operator with rapidly oscillating coefficients, $L_{\varepsilon}$ is given by

$$
L_{\varepsilon}(\cdot)=\frac{1}{2} \sum_{i, j=1}^{d} a_{i j}\left(\frac{x}{\varepsilon}\right) \partial_{x_{i} x_{j}}^{2} \cdot+\sum_{i=1}^{d}\left[\frac{1}{\varepsilon} b_{i}\left(\frac{x}{\varepsilon}\right)+c_{i}\left(\frac{x}{\varepsilon}\right)\right] \partial_{x_{i}} \cdot
$$

$a, b, c$ being periodic functions $\left(a=\sigma \sigma^{*}\right.$ for some periodic function $\sigma$ ).

The paper is organized as follows. Section 2 contains our main assumptions, some preliminary results including ergodicity. In section 3 we prove our main theorem, while the proof of several technical results is delayed until section 4 .

\section{Diffusions with periodic coefficients}

In all what follows, we assume given a complete stochastic basis $\left(\Omega, \mathcal{F},\left(\mathcal{F}_{t}\right)_{0 \leq t \leq T}, \mathbf{P}\right)$, where the filtration $\left(\mathcal{F}_{t}\right)_{0 \leq t \leq T}$ is generated by a $d$-dimensional Brownian motion $\left(B_{t}\right)_{0 \leq t \leq T}$ and the continuous functions

$$
b, c: \mathbb{R}^{d} \longrightarrow \mathbb{R}^{d}, \quad \sigma: \mathbb{R}^{d} \longrightarrow \mathbb{R}^{d} \times \mathbb{R}^{d}
$$

are periodic of period 1 in each direction of $\mathbb{R}^{d}$.

Given $\varepsilon>0, x \in \mathbb{R}^{d}$, let $\left(X_{t}^{x, \varepsilon}\right)_{t \geq 0}$ (which will be mostly written $X_{t}^{\varepsilon}$ ) denote the solution of the stochastic differential equation

$$
\forall t \geq 0, \quad X_{t}^{\varepsilon}=x+\int_{0}^{t}\left[\frac{1}{\varepsilon} b\left(\frac{X_{s}^{\varepsilon}}{\varepsilon}\right)+c\left(\frac{X_{s}^{\varepsilon}}{\varepsilon}\right)\right] d s+\sum_{j=1}^{d} \int_{0}^{t} \sigma_{j}\left(\frac{X_{s}^{\varepsilon}}{\varepsilon}\right) d B_{s}^{j}
$$

and

$$
L_{\varepsilon}(\cdot)=\frac{1}{2} \sum_{i, j=1}^{d} a_{i j}\left(\frac{x}{\varepsilon}\right) \partial_{x_{i} x_{j}}^{2} \cdot+\sum_{i=1}^{d}\left[\frac{1}{\varepsilon} b_{i}\left(\frac{x}{\varepsilon}\right)+c_{i}\left(\frac{x}{\varepsilon}\right)\right] \partial_{x_{i}} .
$$

its infinitesimal generator where for every $x \in \mathbb{R}^{d}, \quad a(x)=\sigma(x) \sigma^{*}(x)$. Considering the processes $\left(\widetilde{X}_{t}^{\varepsilon}\right)_{t \geq 0}$ and $\left(\bar{X}_{t}^{\varepsilon}\right)_{t \geq 0}$ defined by

$$
\forall t \geq 0, \quad \widetilde{X}_{t}^{\varepsilon}=\frac{1}{\varepsilon} X_{\varepsilon^{2} t}^{\varepsilon} \quad ; \quad \bar{X}_{t}^{\varepsilon}=\frac{X_{t}^{\varepsilon}}{\varepsilon}=\widetilde{X}_{t / \varepsilon^{2}}^{\varepsilon}
$$


then there exists a standard $d$-dimensional Brownian motion $\left(B_{t}\right)_{t \geq 0}$ depending on $\varepsilon$ (in fact $B_{s}^{\varepsilon}=\frac{1}{\varepsilon} B_{\varepsilon^{2} s}$ and we forget that dependence), such that for all $t \geq 0$,

$$
\widetilde{X}_{t}^{\varepsilon}=\frac{x}{\varepsilon}+\int_{0}^{t}\left[b\left(\widetilde{X}_{s}^{\varepsilon}\right)+\varepsilon c\left(\widetilde{X}_{s}^{\varepsilon}\right)\right] d s+\sum_{j=1}^{d} \int_{0}^{t} \sigma_{j}\left(\widetilde{X}_{s}^{\varepsilon}\right) d B_{s}^{j} .
$$

We consider the Markov process $\left(\tilde{X}_{t}^{\varepsilon}\right)_{t \geq 0}$ solution of $(2.2)$ as taking values in the $d$ dimensional torus $\mathbf{T}^{\mathbf{d}}=\mathbb{R}^{d} / \mathbb{Z}^{d}$ and denote by $p^{\varepsilon}(t, x, A)$ its transition probability. We shall write $p(t, x, A)$ for $p_{0}(t, x, A)$.

We will also consider the same equation starting from $x$ but without the term $\varepsilon c$, namely

$$
\forall t \geq 0, \quad \widetilde{X}_{t}^{x}=x+\int_{0}^{t} b\left(\tilde{X}_{s}^{x}\right) d s+\sum_{j=1}^{d} \int_{0}^{t} \sigma_{j}\left(\tilde{X}_{s}^{x}\right) d B_{s}^{j}
$$

and let $\left(J_{t}^{x}\right)_{t \geq 0}$ denote the Jacobian of the stochastic flow associated to $\left(\tilde{X}_{t}^{x}\right)_{t \geq 0}$, that is the $d \times d$ matrix valued stochastic process solving

$$
d J_{t}^{x}=D b\left(\widetilde{X}_{t}^{x}\right) J_{t}^{x} d t+\sum_{j=1}^{d} D \sigma_{j}\left(\widetilde{X}_{t}^{x}\right) J_{t}^{x} d B_{t}^{j}, \quad J_{0}^{x}=I .
$$

Moreover to the stochastic differential equation satisfied by $\left(\widetilde{X}_{t}^{x}\right)_{t \geq 0}$, having in mind StroockVaradhan's support theorem, we associate the following controlled ODE (where we use the convention of summation over repeated indices). For each $x \in \mathbf{T}^{\mathbf{d}}, \quad u \in L_{\text {loc }}^{2}\left(\mathbb{R}_{+}, \mathbb{R}^{d}\right)$, $\left(z_{u}^{x, \varepsilon}(t), t \geq 0\right)$ denotes the solution of

$$
\left\{\begin{aligned}
\frac{d z_{i}}{d t}(t) & =\left(b_{i}+\varepsilon c_{i}\right)(z(t))-\frac{1}{2}\left(\partial_{x_{k}} \sigma_{i j} \sigma_{k j}\right)(z(t))+\sigma_{i j}(z(t)) u_{j}(t) \\
z(0) & =x
\end{aligned}\right.
$$

\subsection{Assumptions and preliminary results}

Let us recall the following

Definition 2.1. Consider $b$ and the columns vectors $\sigma_{j}$ of $\sigma$ as vector fields on the torus $\mathbf{T}^{\mathbf{d}}$. We will say that the strong Hörmander condition holds at some point $x \in \mathbf{T}^{\mathbf{d}}$ if the Lie algebra generated by $\left\{\sigma_{j}(x)\right\}_{1 \leq j \leq d}$ spans the whole tangent space of $\mathbf{T}^{\mathbf{d}}$ at $x$.

We furthermore say that the parabolic Hörmander condition holds at $x$, if the Lie algebra generated by the $(d+1)$-dimensional vectors $(b, 1) \cup\left\{\left(\sigma_{j}, 0\right)\right\}_{1 \leq j \leq d}$ spans the whole space $\mathbb{R}^{d+1}$ at $(x, 0) \in \mathbf{T}^{\mathbf{d}} \times \mathbb{R}$.

We say that the drift and the diffusion coefficients satisfy the assumption (H1) ((H1.1) to (H1.5)) if the following holds 
(H1.1) $\sigma, b$ and $c$ are of class $\mathcal{C}^{\infty}$.

(H1.2) There exists a non empty, open and connected subset $U$ of $\mathbf{T}^{\mathbf{d}}$ on which the strong Hörmander conditions holds. Futhermore, there exists $t_{0}$ and $\varepsilon_{0}$ such that

$$
\forall x \in \mathbf{T}^{\mathbf{d}}, \quad 0 \leq \varepsilon \leq \varepsilon_{0}, \quad \inf _{u \in L^{2}\left(0, t_{0}, \mathbb{R}^{d}\right)}\left\{\|u\|_{L^{2}} ; z_{u}^{x, \varepsilon}\left(t_{0}\right) \in U\right\} \quad<\infty .
$$

(H1.3) If $V$ denotes the subset of $\mathbf{T}^{\mathbf{d}}$ where the parabolic Hörmander condition holds, $\tau_{V}^{x}$ the first hitting time of $V$ by the process $\left\{\widetilde{X}_{t}^{x}\right\}$, then

$$
\inf _{t>0} \sup _{x \in \mathbf{T}^{\mathbf{d}}} \mathbf{E}\left(\left|J_{t}^{x}\right|,\left\{\tau_{V}^{x} \geq t\right\}\right)<1 .
$$

It is not difficult to verify that under (H1.1) and (H1.2) the following Doeblin condition is satisfied : there exists $t_{1}>0,0<\varepsilon_{1}<\varepsilon_{0}, \beta>0$ and $\nu$ a probability measure on $\mathbf{T}^{\mathbf{d}}$ which is absolutely continuous with respect to the Lebesgue measure, s.t. for all $0<\varepsilon<\varepsilon_{1}, x \in \mathbf{T}^{\mathbf{d}}$, $A$ a Borel subset of $\mathbf{T}^{\mathbf{d}}$,

$$
p_{\varepsilon}\left(t_{1} ; x, A\right) \geq \beta \nu(A) .
$$

This ensures existence and uniqueness of a unique invariant measure $\mu_{\varepsilon}$ of $\left(\widetilde{X}_{t}^{\varepsilon}\right)_{t \geq 0}$ (we shall write $\mu$ for $\mu_{0}$ ) and the following facts (see [8])

Lemma 2.2 (The spectral gap). There exists $C, \rho>0$ such that for all $0 \leq \varepsilon \leq 1, t \geq 0$ and $f \in L^{\infty}\left(\mathbf{T}^{\mathrm{d}}\right)$,

$$
\left|\mathbf{E} f\left(\widetilde{X}_{t}^{\varepsilon}\right)-\int_{\mathbf{T}^{\mathbf{d}}} f(x) \mu_{\varepsilon}(d x)\right| \leq C\|f\|_{\infty} e^{-\rho t}
$$

Lemma 2.3. The following holds

$$
\mu_{\varepsilon} \stackrel{\varepsilon \longrightarrow 0}{\longrightarrow} \mu, \quad \text { weakly }
$$

As a consequence we have the following sort of ergodic theorem

Corollary 2.4. Let $f \in L^{\infty}\left(\mathbf{T}^{\mathbf{d}}\right)$. Then for any $t \geq 0$,

$$
\int_{0}^{t} f\left(\bar{X}_{s}^{\varepsilon, x}\right) d s \rightarrow t \int_{\mathbf{T}^{\mathbf{d}}} f(y) \mu(d y)
$$

We finally assume that

(H1.4) The crucial centering condition is satisfied : $\int_{\mathbf{T}^{\mathbf{d}}} b(x) \mu(d x)=0$. 


\subsection{The Poisson equation}

Let us consider the infinitesimal generator $L$ of the $\mathbf{T}^{\mathbf{d}}$-valued diffusion process $\left(\widetilde{X}_{t}^{x}\right)_{t \geq 0}$ given by

$$
L=\frac{1}{2} \sum_{i, j=1}^{d}\left(\sigma \sigma^{*}\right)_{i j}(x) \partial_{x_{i} x_{j}}^{2}+\sum_{i=1}^{d} b_{i}(x) \partial_{x_{i}}
$$

and $\mathcal{P}_{t}$ the semigroup generated by $\left(\widetilde{X}_{t}^{x}\right)_{t \geq 0}$.

For some functions $f \in \mathcal{C}^{1}\left(\mathbf{T}^{\mathbf{d}}\right)$ satisfying the centering condition

$$
\int_{\mathbf{T}^{\mathbf{d}}} f(x) \mu(d x)=0,
$$

we want to solve the PDE

$$
L \widehat{f}(x)+f(x)=0, \quad x \in \mathbf{T}^{\mathbf{d}} .
$$

This will be essential in order to get rid of the terms depending on $\varepsilon^{-1}$ in the perturbed equations. For this purpose we recall the following result given in [8, Lemma 2.6] which will be useful in the sequel :

Lemma 2.5. Under $(\mathbf{H 1 . 1})-(\mathbf{H 1} \mathbf{1 . 3}), \mathcal{P}_{t}$ maps $\mathcal{C}^{1}\left(\mathbf{T}^{\mathbf{d}}\right)$ into itself and there exists two positive constants $K>0$ and $\rho>0$ such that for every $f \in \mathcal{C}^{1}\left(\mathbf{T}^{\mathbf{d}}\right)$ satisfying (2.7) and for every $t \geq 0$, we have

$$
\left\|\mathcal{P}_{t} f\right\|_{\mathcal{C}^{1}\left(\mathbf{T}^{\mathbf{d}}\right)} \leq K e^{-\rho t}\|f\|_{\mathcal{C}^{1}\left(\mathbf{T}^{\mathbf{d}}\right)}
$$

It follows from Lemma 2.5 the

Lemma 2.6. Under (H1.1)-(H1.3), if $f \in \mathcal{C}^{1}\left(\mathbf{T}^{\mathbf{d}}\right)$ satisfies (2.7), then the function $\widehat{f}$ defined by

$$
\widehat{f}(x)=\int_{0}^{+\infty} \mathbf{E}_{x}\left[f\left(\widetilde{X}_{t}\right)\right] d t, \quad x \in \mathbf{T}^{\mathbf{d}},
$$

belongs to $\mathcal{C}^{1}\left(\mathbf{T}^{\mathbf{d}}\right)$ and is the unique weak sense solution of equation (2.8) which is centered with respect to $\mu$.

For the notion of weak sense solution to (2.8), see [16]. Under the previous assumptions, for $i=1, \ldots, d$, we can consider the following Poisson equation on the torus $\mathbf{T}^{\mathbf{d}}$ :

$$
\widehat{L b}_{i}(\cdot)+b_{i}(\cdot)=0
$$

Thanks to Lemma 2.5, for any $i=1, \ldots, d$, the function $\widehat{b}_{i}$ solution of $(2.10)$ belongs to $\mathcal{C}^{1}\left(\mathbf{T}^{\mathbf{d}}\right)$ and is given for any $x \in \mathbf{T}^{\mathbf{d}}$ by

$$
\widehat{b}_{i}(x)=\int_{0}^{+\infty} \mathbf{E}_{x}\left[b_{i}\left(\widetilde{X}_{t}\right)\right] d t .
$$


Let us consider the constant coefficients $A$ and $C$ given by $\left(\right.$ with $\left.\Lambda(x)=\left(I+\partial_{x} \widehat{b}\right)(x) \sigma(x)\right)$

$$
A=\int_{\mathbf{T}^{\mathbf{d}}}\left(\Lambda \Lambda^{*}\right)(x) \mu(d x) ; \quad C=\int_{\mathbf{T}^{\mathbf{d}}}\left(I+\partial_{x} \widehat{b}\right) c(x) \mu(d x)
$$

and the diffusion $\left(X_{t}^{x}\right)_{t \geq 0}$ given by

$$
\forall t \geq 0, \quad X_{t}^{x}=x+C t+A^{1 / 2} B_{t}
$$

We state the following crucial condition

(H1.5) The matrix $A$ is positive definite.

Remark : Necessary and sufficient condition for (H1.5) to hold are given in [8] in terms of the diffusion $\left(\widetilde{X}_{t}\right)_{t \geq 0}$ and the support of its invariant measure.

Recall the subset $G \subset \mathbb{R}^{d}$ from the Introduction. Define the stopping times $\tau_{x}^{\varepsilon}=\inf \{t \geq$ $\left.0, X_{t}^{\varepsilon, x} \notin \bar{G}\right\}$ and $\tau_{x}=\inf \left\{t \geq 0, X_{t}^{x} \notin \bar{G}\right\}$ (the subscript $x$ will be often omitted for notational simplicity). Note that (H1.5) implies that $\tau_{x}=0$, a.s. for all $x \in \partial G$.

We have the following result established in [8, Theorem 3.1]

Proposition 2.7. Under assumptions (H1), the following weak convergence holds

$$
\left(X^{x, \varepsilon}, \tau_{x}^{\varepsilon}\right) \Longrightarrow\left(X^{x}, \tau_{x}\right) \quad \text { in } \quad \mathcal{C}\left(\mathbb{R}_{+}, \bar{G}\right) \times \mathbb{R}_{+} .
$$

We are now in position to study our main subject.

\section{Homogenization of an elliptic PDE}

For each $\varepsilon>0$, we consider the elliptic PDE with Dirichlet boundary condition

$$
\left\{\begin{array}{l}
L_{\varepsilon} u^{\varepsilon}(x)+f\left(\frac{x}{\varepsilon}, x, u^{\varepsilon}(x), \partial_{x} u^{\varepsilon}(x) \sigma\left(\frac{x}{\varepsilon}\right)\right)=0, \quad x \in G, \\
u^{\varepsilon}(x)=g(x), \quad x \in \partial G
\end{array}\right.
$$

where $G \subset \mathbb{R}^{d}$ is a smooth bounded domain whose boundary is of class $\mathcal{C}^{2}, g \in \mathcal{C}^{2}(\partial G)$ and $f: \mathbb{R}^{d} \times \mathbb{R}^{d} \times \mathbb{R} \times \mathbb{R}^{d} \longrightarrow \mathbb{R}$ is continuous and satisfies the following assumptions (H2) (in what follows, the reader should keep in mind that $y$ stands for $u^{\varepsilon}$ and $z$ for $\partial_{x} u^{\varepsilon}$ ) :

(H2.1) $f$ is periodic of period one in each direction of $\mathbb{R}^{d}$ in the first argument.

(H2.2) There exists two constants $\mu<0$ and $K>0$ such that for every $x \in \mathbf{T}^{\mathbf{d}}, \quad\left(\widetilde{x}, \widetilde{x}^{\prime}\right) \in$ $\mathbb{R}^{d} \times \mathbb{R}^{d},\left(y, y^{\prime}\right) \in \mathbb{R}^{2}$ and $\left(z, z^{\prime}\right) \in\left(\mathbb{R}^{d}\right)^{2}$,

$$
\begin{aligned}
\left(y-y^{\prime}\right)(f(x, \widetilde{x}, y, z)- & \left.f\left(x, \widetilde{x}, y^{\prime}, z\right)\right) \leq \mu\left(y-y^{\prime}\right)^{2} \\
\left|f(x, \widetilde{x}, y, z)-f\left(x, \widetilde{x}, y, z^{\prime}\right)\right| & \leq K|| z-z^{\prime}|| \\
|f(x, \widetilde{x}, y, z)| & \leq K(1+|y|+|z|)
\end{aligned}
$$


(H2.3) There exists a function $\varphi \in \mathcal{C}\left(\mathbb{R}_{+}, \mathbb{R}_{+}\right)$satisfying $\varphi(0)=0$ such that for every $x \in \mathbf{T}^{\mathbf{d}}, \quad\left(\widetilde{x}, \widetilde{x}^{\prime}\right) \in \mathbb{R}^{d} \times \mathbb{R}^{d},(y, z) \in \mathbb{R} \times \mathbb{R}^{d}$,

$$
\left|f(x, \widetilde{x}, y, z)-f\left(x, \widetilde{x}^{\prime}, y, z\right)\right| \leq \varphi\left(\left|\widetilde{x}-\widetilde{x}^{\prime}\right|(1+|z|)\right) .
$$

(H2.4) There exists a constant $\lambda \neq 0$ such that $\lambda>2 \mu+K^{2}$ and

$$
\sup _{x \in \bar{G}} \sup _{\varepsilon>0} \mathbf{E}_{x} e^{\lambda \tau^{\varepsilon}}<\infty .
$$

(H2.5) For every $\varepsilon>0$, the set $\Gamma^{\varepsilon}=\left\{x \in \partial G: \mathbf{P}\left(\tau_{x}^{\varepsilon}>0\right)=0\right\}$ is closed.

Let us consider the second order differential operator

$$
\bar{L}=\frac{1}{2} \sum_{i, j=1}^{d} A_{i j} \partial_{x_{i} x_{j}}^{2}+\sum_{i=1}^{d} C_{i} \partial_{x_{i}} .
$$

We are interested in the elliptic PDE

$$
\left\{\begin{array}{l}
\bar{L} u(x)+\bar{f}\left(x, u(x), \partial_{x} u(x)\right)=0, \quad x \in G, \\
u(x)=g(x), \quad x \in \partial G .
\end{array}\right.
$$

where the function $\bar{f}$ is given by (recall that $\left.\Lambda(x)=\left(I+\partial_{x} \hat{b}\right)(x) \sigma(x)\right)$

$$
\forall(\widetilde{x}, y, z) \in \mathbb{R}^{d} \times \mathbb{R} \times \mathbb{R}^{d}, \quad \bar{f}(\widetilde{x}, y, z)=\int_{\mathbf{T}^{\mathbf{d}}} f(x, \widetilde{x}, y, \Lambda(x) z) \mu(d x) .
$$

It is easy to see that $\bar{f}$ is jointly continuous and satisfies assumption (H2.2). So using the BSDE with random terminal time

$$
Y_{t \wedge \tau}^{x}=g\left(X_{\tau}^{x}\right)+\int_{t \wedge \tau}^{\tau} \bar{f}\left(X_{r}^{x}, Y_{r}^{x}, Z_{r}^{x}\right) d r-\int_{t \wedge \tau}^{\tau} Z_{r}^{x} d B_{r}, \quad t \geq 0,
$$

we deduce thanks to [15, Corollary 6.96], that under our standing assumptions, $u(x)=Y_{0}^{x} \in$ $\mathcal{C}(\bar{G})$ and is the unique viscosity solution of (3.4).

We now formulate our main result :

Theorem 3.1. Under assumptions $(\mathbf{H 1})$ and $(\mathbf{H 2})$, for all $x \in G$, we have

$$
u^{\varepsilon}(x) \stackrel{\varepsilon \longrightarrow 0}{\longrightarrow} u(x) .
$$

Before proving Theorem 3.1, let us establish the following technical result to face the lack of smoothness of $u$ (whose proof is given in section 4). 
Proposition 3.2. Assume that (H1) and (H2) are in force. Then there exists a sequence of functions $\left(u^{n}\right)_{n \geq 1} \subset \mathcal{C}^{\infty}\left(\mathbb{R}^{d}\right)$ satisfying :

(i) There exists a constant $\Gamma>0$ such that

$$
\forall n \geq 1, \quad \forall x \in G, \quad\left|\partial_{x} u^{n}\right|+\left|u^{n}(x)\right| \leq \Gamma .
$$

(ii) $\sup _{x \in \bar{G}}\left[\left|u^{n}(x)-u(x)\right|+\left|\partial u^{n}(x)-\partial u(x)\right|\right] \rightarrow 0, \quad$ as $n \rightarrow \infty$.

(iii) The sequence $\left(u^{n}\right)_{n \geq 1}$ satisfies $\bar{L} u^{n}(x)+\bar{f}\left(x, u^{n}(x), \partial_{x} u^{n}(x)\right) \rightarrow 0$ as $n \rightarrow \infty$ uniformly on $G^{\delta}=\left\{x \in G: d\left(x, G^{c}\right) \geq \delta\right\}$, for any $\delta>0$.

(iv) For any $p \geq 1, \quad \bar{L} u^{n}+\bar{f}\left(\cdot, u^{n}(\cdot), \partial_{x} u^{n}(\cdot)\right)$ is uniformly bounded in $L^{p}(G)$.

\subsection{The homogenization property}

Our approach to prove our main result is purely probabilistic and is based on BSDE techniques. The strategy consists in introducing the unique pair $\left(Y_{t}^{\varepsilon, x}, Z_{t}^{\varepsilon, x}\right)_{0 \leq t \leq \tau^{\varepsilon}}$ of $\mathcal{F}_{t^{-}}$ progressively measurable processes solution of the BDSE with random terminal time

$$
\forall t \geq 0, \quad Y_{t \wedge \tau^{\varepsilon}}^{\varepsilon, x}=g\left(X_{\tau^{\varepsilon}}^{\varepsilon, x}\right)+\int_{t \wedge \tau^{\varepsilon}}^{\tau^{\varepsilon}} f\left(\bar{X}_{r}^{\varepsilon, x}, X_{r}^{\varepsilon, x}, \quad Y_{r}^{\varepsilon, x}, Z_{r}^{\varepsilon, x}\right) d r-\int_{t \wedge \tau^{\varepsilon}}^{\tau^{\varepsilon}} Z_{r}^{\varepsilon, x} d B_{r}
$$

satisfying

$$
\mathbf{E}\left(\sup _{0 \leq t \leq \tau^{\varepsilon}} e^{\lambda t}\left|Y_{t}^{\varepsilon, x}\right|^{2}+\int_{0}^{\tau^{\varepsilon}} e^{\lambda t}\left|Z_{t}^{\varepsilon, x}\right|^{2} d t\right)<\infty .
$$

It is well known (see [13, Theorem 5.3]) that the function $u^{\varepsilon}(x)=Y_{0}^{\varepsilon, x}$ is a viscosity solution of (3.1).

Let us consider the process $M_{t}^{\varepsilon}=-\int_{0}^{t} Z_{r}^{\varepsilon, x} d B_{r}, t \geq 0$. We intend to study the tightness property of the pair of processes $\left(Y_{*}^{\varepsilon}, M_{*}^{\varepsilon}\right)$ indexed by $\varepsilon>0$ in the space $\mathcal{D}\left(\mathbb{R}_{+} ; \mathbb{R}^{d+1}\right)$ (the space of right continuous functions having left limits) equipped with the $S$-topology of Jakubowski (see [11] for further details).

For this end it suffices to establish this result on the interval $[0, T]$ for every $T>0$.

Let us recall that the sequence of quasi-martingales $\left\{U_{s}^{n} ; 0 \leq s \leq T\right\}$ defined on the filtered probability space $\left\{\Omega ; \mathcal{F},\left(\mathcal{F}_{s}\right)_{0 \leq s \leq T}, \mathbf{P}\right\}$ is tight whenever

$$
\sup _{n}\left[\sup _{0 \leq s \leq T} \mathbf{E}\left|U_{s}^{n}\right|+C V_{T}^{0}\left(U^{n}\right)\right]<\infty,
$$

where $C V_{T}^{0}\left(U^{n}\right)$, the so-called "conditional variation of $U^{n}$ on $[0, T]$ ", is defined as

$$
C V_{T}^{0}\left(U^{n}\right)=\sup \mathbf{E}\left(\sum_{i=1}\left|\mathbf{E}\left(U_{t_{i+1}}^{n}-U_{t_{i}}^{n} \mid \mathcal{F}_{t_{i}}\right)\right|\right)
$$

and the supremum is taken over all partitions of the interval $[0, T]$.

We claim that (the proof is given in section 4) 
Proposition 3.3. There exists a positive constant $C_{3.3}>0$ such that

$$
\sup _{\varepsilon>0} \mathbf{E}\left[\sup _{0 \leq r \leq \tau^{\varepsilon}} e^{\lambda r}\left|Y_{r}^{\varepsilon}\right|^{2}+\int_{0}^{\tau^{\varepsilon}} e^{\lambda r}\left|Z_{r}^{\varepsilon}\right|^{2} d s\right] \leq C_{3.3}
$$

As a consequence, we have

Corollary 3.4. For any $T>0$, the family of processes $\left(Y^{\varepsilon}, M^{\varepsilon}\right)$ indexed by $\varepsilon>0$ is $\mathbf{P}-$ tight as elements of $\mathcal{D}\left([0, T], \mathbb{R}^{d+1}\right)$, equipped with the $S$-topology of Jakubowski.

To deal with the highly oscillating terms (depending on $\varepsilon^{-1}$ ) in the diffusion (2.1), we consider the process $\left(\widehat{X}_{t}^{\varepsilon}\right)_{t \geq 0}\left(\right.$ recall that $\left.\bar{X}_{t}^{\varepsilon}=X_{t}^{\varepsilon} / \varepsilon\right)$ given by

$$
\forall t \geq 0, \quad \widehat{X}_{t}^{\varepsilon}=X_{t}^{\varepsilon}+\varepsilon\left(\widehat{b}\left(\bar{X}_{t}^{\varepsilon}\right)-\widehat{b}\left(\frac{x}{\varepsilon}\right)\right)
$$

Thanks to Itô's formula (see [8, Lemma 3.2]), we have

$$
\forall t \geq 0, \quad \widehat{X}_{t}^{\varepsilon}=x+\int_{0}^{t}\left(I+\partial_{x} \hat{b}\right) c\left(\bar{X}_{r}^{\varepsilon}\right) d r+\int_{0}^{t} \Lambda\left(\bar{X}_{r}^{\varepsilon}\right) d B_{r} .
$$

As a consequence we deduce that the sequence of processes $\left\{X_{s}^{\varepsilon}, 0 \leq s \leq t, 0<\varepsilon \leq\right.$ $1\}$ is tight in the space $\mathcal{C}\left([0, t], \mathbb{R}^{d}\right)$ endowed with the topology of uniform convergence. Moreover thanks to the martingale central limit theorem [5, Theorem 7.1.4] which follows from Corollary 2.4, we have

$$
\int_{0}^{\cdot} \Lambda\left(\bar{X}_{s}^{\varepsilon}\right) d B_{s} \Longrightarrow A^{1 / 2} B . \quad \text { in } \mathcal{C}\left([0, T] ; \mathbb{R}^{d}\right) \quad \text { as } \quad \varepsilon \rightarrow 0 .
$$

Hence there exists a subsequence (still note as the whole sequence) such that

$$
\left(X^{\varepsilon}, Y^{\varepsilon}, M^{\varepsilon}\right) \Longrightarrow(X, Y, M) \quad \text { in } \mathcal{D}\left([0, T] ; \mathbb{R}^{2 d+1}\right) .
$$

Let us assume that the following extension of Corollary 2.4 holds (the proof is given in section 4).

Proposition 3.5. Let $\Psi: \mathbb{R}^{d} \times \mathbb{R}^{N} \rightarrow \mathbb{R}$ be a measurable function, periodic with respect to its first variable, satisfying:

i) for any $R>0$, we can find $K_{R}>0$ such that whenever $\left(x, v, v^{\prime}\right) \in \mathbb{R}^{d} \times \mathbb{R}^{N} \times \mathbb{R}^{N},|v| \leq R$ and $\left|v^{\prime}\right| \leq R$, then we have $\left|\Psi(x, v)-\Psi\left(x, v^{\prime}\right)\right| \leq K_{R}\left|v-v^{\prime}\right|$.

ii) there exists $M>0$ such that for any $x \in \mathbb{R}^{d}, v \in \mathbb{R}^{N}, \quad|\Psi(x, v)| \leq M(1+|v|)$.

Suppose additionally that $\left(V^{\varepsilon}\right)_{\varepsilon>0}$ is a family of $\mathbb{R}^{N}$-valued processes, which is tight in $\mathcal{D}\left([0, T] ; \mathbb{R}^{N}\right)$ equipped with the $S$-topology of Jakubowski and satisfies

$$
\sup _{\varepsilon>0} \mathbf{E}\left(\sup _{0 \leq s \leq \tau^{\varepsilon}} e^{\lambda s}\left|V_{s}^{\varepsilon}\right|^{2}\right)<\infty
$$


Then the following convergence holds: for any $\nu<\lambda$,

$$
\mathbf{E}\left|\int_{0}^{\tau^{\varepsilon}} e^{\nu r} \Psi\left(\bar{X}_{r}^{\varepsilon}, V_{r}^{\varepsilon}\right) d r-\int_{0}^{\tau^{\varepsilon}} e^{\nu r} \bar{\Psi}\left(V_{r}^{\varepsilon}\right) d r\right| \rightarrow 0, \quad \text { as } \varepsilon \text { tends to } 0
$$

where $\bar{\Psi}(v)=\int_{\mathbf{T}^{\mathrm{d}}} \Psi(x, v) \mu(d x)$.

From now on our strategy consists in proving that the difference $Y_{0}^{\varepsilon, x}-u(x)$ tends to 0 as $\varepsilon$ goes to 0 . However in the following computations, we are faced with the lack of smoothness of the function $u$. To overcome the difficulty, we approximate the function $u$ with the help of the smooth sequence $\left(u^{n}\right)_{n \in \mathbb{N}}$ defined in Proposition 3.2. Thus we consider, for every $n \in \mathbb{N}$ the pair of processes $\left(\widetilde{Y}_{s}^{\varepsilon, n}, \widetilde{Z}_{s}^{\varepsilon, n}\right)_{s \geq 0}$ defined by

$$
\forall s \geq 0, \quad \tilde{Y}_{s}^{\varepsilon, n}=Y_{s}^{\varepsilon, x}-u^{n}\left(\widehat{X}_{s \wedge \tau^{\varepsilon}}^{\varepsilon}\right) ; \quad \widetilde{Z}_{s}^{\varepsilon, n}=Z_{s}^{\varepsilon, x}-\partial_{x} u^{n}\left(\widehat{X}_{s}^{\varepsilon}\right) \Lambda\left(\bar{X}_{s}^{\varepsilon}\right) .
$$

Our main result, Theorem 3.1, is an immediate consequence of

Theorem 3.6. The following holds

(i) There exists a constant $C_{3.6}>0$ such that for every $\varepsilon>0, n \in \mathbb{N}$ and $t \geq 0$, we have

$$
\left|\tilde{Y}_{t}^{\varepsilon, n}\right| \leq C_{3.6} \quad \text { a.s. }
$$

(ii) For all $\eta>0$ there exists an integer $n(\eta)$ such that for all $n \geq n(\eta)$,

$$
\limsup _{\varepsilon \longrightarrow 0}\left|\widetilde{Y}_{0}^{\varepsilon, n}\right| \leq \eta
$$

Proof. Step 1 : Proof of (i). Itô's formula applied to the function $(t, y) \mapsto e^{\lambda t} y^{2}$ yields that for every $t \geq 0$

$$
\begin{aligned}
e^{\lambda t \wedge \tau^{\varepsilon}}\left|Y_{t \wedge \tau^{\varepsilon}}^{\varepsilon, x}\right|^{2}+ & \int_{t \wedge \tau^{\varepsilon}}^{\tau^{\varepsilon}} e^{\lambda r}\left|Z_{r}^{\varepsilon, x}\right|^{2} d r=e^{\lambda \tau^{\varepsilon}}\left|g\left(X_{\tau^{\varepsilon}}^{\varepsilon}\right)\right|^{2}-\int_{t \wedge \tau^{\varepsilon}}^{\tau^{\varepsilon}} \lambda e^{\lambda r}\left|Y_{r}^{\varepsilon, x}\right|^{2} d r \\
& +2 \int_{t \wedge \tau^{\varepsilon}}^{\tau^{\varepsilon}} e^{\lambda r} Y_{r}^{\varepsilon, x} f(\Theta(\varepsilon, r)) d r-2 \int_{t \wedge \tau^{\varepsilon}}^{\tau^{\varepsilon}} e^{\lambda r} Y_{r}^{\varepsilon, x} Z_{r}^{\varepsilon, x} d B_{r},
\end{aligned}
$$

where for every $\varepsilon>0$ and $r>0, \Theta(\varepsilon, r)=\left(\bar{X}_{r}^{\varepsilon, x}, X_{r}^{\varepsilon, x}, Y_{r}^{\varepsilon, x}, Z_{r}^{\varepsilon, x}\right)$.

Using standard estimates and assumptions (H2.2), we have

$2 e^{\lambda r} Y_{r}^{\varepsilon, x} f(\Theta(\varepsilon, r)) \leq e^{\lambda r}\left(K^{2}+2 \mu\right)\left|Y_{r}^{\varepsilon, x}\right|^{2}+e^{\lambda r}\left|Z_{r}^{\varepsilon, x}\right|^{2}+\alpha e^{\lambda r}\left|Y_{r}^{\varepsilon, x}\right|^{2}+\frac{1}{\alpha} e^{\lambda r}\left|f\left(\bar{X}_{r}^{\varepsilon, x}, X_{r}^{\varepsilon, x}, 0,0\right)\right|^{2}$

with $\alpha=\lambda-\left(2 \mu+K^{2}\right)>0$. Since $g$ is bounded, there exists a positive constant $K^{\prime}$ such that for every $t \geq 0$,

$$
e^{\lambda t \wedge \tau^{\varepsilon}}\left|Y_{t \wedge \tau^{\varepsilon}}^{\varepsilon, x}\right|^{2} \leq K^{\prime} e^{\lambda \tau^{\varepsilon}}+\frac{1}{\alpha} \int_{t \wedge \tau^{\varepsilon}}^{\tau^{\varepsilon}} e^{\lambda r}\left|f\left(\bar{X}_{r}^{\varepsilon, x}, X_{r}^{\varepsilon, x}, 0,0\right)\right|^{2} d r-2 \int_{t \wedge \tau^{\varepsilon}}^{\tau^{\varepsilon}} e^{\lambda r} Y_{r}^{\varepsilon, x} Z_{r}^{\varepsilon, x} d B_{r}
$$


Taking the conditional expectation $\mathbf{E}^{\mathcal{F}_{t}}$, we deduce thanks to assumption (H2.2),

$$
\forall \varepsilon>0, \quad t \geq 0, \quad\left|Y_{t}^{\varepsilon, x}\right|^{2} \leq K^{\prime} \mathbf{E}^{\mathcal{F}_{t}}\left(e^{\lambda\left(\tau^{\varepsilon}-t\right)^{+}}\right)
$$

From the Markov property, the term of the right hand side is equal to $\mathbf{E}_{X_{t}^{\varepsilon}}\left(e^{\lambda \tau^{\varepsilon}}\right)$. Hence from (3.2) there exists a constant $C_{3.6}>0$ such that

$$
\forall \varepsilon>0, \quad t \geq 0, \quad\left|Y_{t}^{\varepsilon, x}\right|^{2} \leq C_{3.6}
$$

(i) follows, since $u^{n}$ is bounded, uniformly w. r. t. $n \geq 1$.

STEP 2 : AN UPPER BOUND FOR $\tilde{Y}_{0}^{\varepsilon, n}$. Note that for every $s \geq 0$,

$$
\widehat{X}_{s \wedge \tau^{\varepsilon}}=\widehat{X}_{\tau^{\varepsilon}}^{\varepsilon}-\int_{s \wedge \tau^{\varepsilon}}^{\tau^{\varepsilon}}\left(I+\partial_{x} \widehat{b}\right) c\left(\bar{X}_{s}^{\varepsilon}\right) d r-\int_{s \wedge \tau^{\varepsilon}}^{\tau^{\varepsilon}} \Lambda\left(\bar{X}_{r}^{\varepsilon}\right) d B_{r}
$$

Since $u^{n} \in C^{\infty}\left(\mathbb{R}^{d}\right)$, Itô's Formula yields for any $s \geq 0$,

$$
u^{n}\left(\widehat{X}_{s \wedge \tau^{\varepsilon}}^{\varepsilon}\right)=u^{n}\left(\widehat{X}_{\tau^{\varepsilon}}^{\varepsilon}\right)-\int_{s \wedge \tau^{\varepsilon}}^{\tau^{\varepsilon}} \hat{L}^{\varepsilon, n}(r) d r-\int_{s \wedge \tau^{\varepsilon}}^{\tau^{\varepsilon}} \partial_{x} u^{n}\left(\widehat{X}_{r}^{\varepsilon}\right) \Lambda\left(\bar{X}_{r}^{\varepsilon}\right) d B_{r},
$$

where we define

$$
\hat{L}^{\varepsilon, n}(s)=\frac{1}{2} \sum_{i, j}\left(\Lambda \Lambda^{*}\right)\left(\bar{X}_{s}^{\varepsilon}\right) \partial_{x_{i} x_{j}} u^{n}\left(\widehat{X}_{s}^{\varepsilon}\right)+\sum_{i}\left[\left(I+\partial_{x} \widehat{b}\right) c\left(\bar{X}_{s}^{\varepsilon}\right)\right] \partial_{x} u^{n}\left(\widehat{X}_{s}^{\varepsilon}\right) .
$$

Putting pieces together, we deduce that for any $s \geq 0$,

$$
\tilde{Y}_{s \wedge \tau^{\varepsilon}}^{\varepsilon, n}=g\left(X_{\tau^{\varepsilon}}^{\varepsilon}\right)-u^{n}\left(\widehat{X}_{\tau^{\varepsilon}}^{\varepsilon}\right)+\int_{s \wedge \tau^{\varepsilon}}^{\tau^{\varepsilon}}\left[f(\Theta(\varepsilon, r))+\hat{L}^{\varepsilon, n}(r)\right] d r-\int_{s \wedge \tau^{\varepsilon}}^{\tau^{\varepsilon}} \widetilde{Z}_{r}^{\varepsilon, n} d B_{r}
$$

Let $\nu=2 \mu+K^{2}$. Itô's formula applied to the function $(t, y) \mapsto e^{\nu t} y^{2}$ yields for $t \geq 0$,

$$
\begin{aligned}
e^{\nu t \wedge \tau^{\varepsilon}}\left|\widetilde{Y}_{t \wedge \tau^{\varepsilon}}^{\varepsilon, n}\right|^{2}+\int_{t \wedge \tau^{\varepsilon}}^{\tau^{\varepsilon}} e^{\nu s}\left|\widetilde{Z}_{s}^{\varepsilon, n}\right|^{2} d s & =e^{\nu \tau^{\varepsilon}}\left|g\left(X_{\tau^{\varepsilon}}^{\varepsilon}\right)-u^{n}\left(\widehat{X}_{\tau^{\varepsilon}}^{\varepsilon}\right)\right|^{2}-\int_{t \wedge \tau^{\varepsilon}}^{\tau^{\varepsilon}} \nu e^{\nu s}\left|\widetilde{Y}_{s}^{\varepsilon, n}\right|^{2} d s \\
& +2 \int_{t \wedge \tau^{\varepsilon}}^{\tau^{\varepsilon}} e^{\nu s} \widetilde{Y}_{s}^{\varepsilon, n}\left[\hat{L}^{\varepsilon, n}(s)+f(\Theta(\varepsilon, s)] d s\right. \\
& -2 \int_{t \wedge \tau^{\varepsilon}}^{\tau^{\varepsilon}} e^{\nu s} \widetilde{Y}_{s}^{\varepsilon, n} \widetilde{Z}_{s}^{\varepsilon, n} d B_{s}
\end{aligned}
$$

Consider the decomposition

$$
\begin{aligned}
\tilde{Y}_{s}^{\varepsilon, n}\left[\hat{L}^{\varepsilon, n}(s)+f(\Theta(\varepsilon, s)]\right. & =\widetilde{Y}_{s}^{\varepsilon, n}\left[\hat{L}^{\varepsilon, n}(s)+f\left(\bar{X}_{s}^{\varepsilon, x}, X_{s}^{\varepsilon, x}, u^{n}\left(\widehat{X}_{s \wedge \tau^{\varepsilon}}^{\varepsilon}\right), \partial_{x} u^{n}\left(\widehat{X}_{s}^{\varepsilon}\right) \Lambda\left(\bar{X}_{s}^{\varepsilon}\right)\right]\right. \\
& +\widetilde{Y}_{s}^{\varepsilon, n}\left[f \left(\Theta(\varepsilon, s)-f\left(\bar{X}_{s}^{\varepsilon, x}, X_{s}^{\varepsilon, x}, u^{n}\left(\widehat{X}_{s \wedge \tau^{\varepsilon}}^{\varepsilon}\right), \partial_{x} u^{n}\left(\widehat{X}_{s}^{\varepsilon}\right) \Lambda\left(\bar{X}_{s}^{\varepsilon}\right)\right] .\right.\right.
\end{aligned}
$$


From assumption (H2.3), the second term of the last right hand side is less than

$$
\mu\left|\widetilde{Y}_{s}^{\varepsilon, n}\right|^{2}+K\left|\widetilde{Y}_{s}^{\varepsilon, n}\right|\left|\widetilde{Z}_{s}^{\varepsilon, n}\right|
$$

Hence using standard estimates, we deduce that

$$
\begin{aligned}
\left|\widetilde{Y}_{0}^{\varepsilon, n}\right|^{2} & \leq \mathbf{E}\left(e^{\nu \tau^{\varepsilon}}\left|g\left(X_{\tau^{\varepsilon}}^{\varepsilon}\right)-u^{n}\left(\widehat{X}_{\tau^{\varepsilon}}^{\varepsilon}\right)\right|^{2}\right)+2 \mathbf{E} \int_{0}^{\tau^{\varepsilon}} e^{\nu s} \widetilde{Y}_{s}^{\varepsilon, n}\left[\delta_{1, n}(\varepsilon, s)+\delta_{2, n}(\varepsilon, s)\right] d s \\
& +2 \mathbf{E} \int_{0}^{\tau^{\varepsilon}} e^{\nu s} \widetilde{Y}_{s}^{\varepsilon, n}\left[\hat{L}^{\varepsilon, n}(s)-\bar{L} u^{n}\left(\widehat{X}_{s}^{\varepsilon}\right)\right] d s
\end{aligned}
$$

with

$$
\begin{aligned}
& \delta_{1, n}(\varepsilon, s)=f\left(\bar{X}_{s}^{\varepsilon, x}, X_{s}^{\varepsilon, x}, u^{n}\left(\widehat{X}_{s}^{\varepsilon}\right), \partial_{x} u^{n}\left(\widehat{X}_{s}^{\varepsilon}\right) \Lambda\left(\bar{X}_{s}^{\varepsilon}\right)\right)-\bar{f}\left(X_{s}^{\varepsilon, x}, u^{n}\left(\widehat{X}_{s}^{\varepsilon}\right), \partial_{x} u^{n}\left(\widehat{X}_{s}^{\varepsilon}\right)\right) \\
& \delta_{2, n}(\varepsilon, s)=\bar{L} u^{n}\left(\widehat{X}_{s}^{\varepsilon}\right)+\bar{f}\left(X_{s}^{\varepsilon, x}, u^{n}\left(\widehat{X}_{s}^{\varepsilon}\right), \partial_{x} u^{n}\left(\widehat{X}_{s}^{\varepsilon}\right)\right) .
\end{aligned}
$$

Let

$$
\begin{aligned}
C_{n}(\varepsilon) & =\mathbf{E}\left(e^{\nu \tau^{\varepsilon}}\left|g\left(X_{\tau^{\varepsilon}}^{\varepsilon}\right)-u^{n}\left(\widehat{X}_{\tau^{\varepsilon}}^{\varepsilon}\right)\right|^{2}\right)+2 \mathbf{E} \int_{0}^{\tau^{\varepsilon}} e^{\nu s} \widetilde{Y}_{s}^{\varepsilon, n}\left[\hat{L}^{\varepsilon, n}(s)-\bar{L} u^{n}\left(\widehat{X}_{s}^{\varepsilon}\right)\right] d s \\
& +2 \mathbf{E} \int_{0}^{\tau^{\varepsilon}} e^{\nu s} \widetilde{Y}_{s}^{\varepsilon, n} \delta_{1, n}(\varepsilon, s) d s+2 \mathbf{E} \int_{0}^{\tau^{\varepsilon}} e^{\nu s} \widetilde{Y}_{s}^{\varepsilon, n} \delta_{2, n}(\varepsilon, s) d s \\
& :=C_{n}(1, \varepsilon)+C_{n}(2, \varepsilon)+C_{n}(3, \varepsilon)+C_{n}(4, \varepsilon) .
\end{aligned}
$$

We have that for every $n \geq 1$ and $\varepsilon>0$,

$$
\left|\tilde{Y}_{0}^{\varepsilon, n}\right|^{2} \leq C_{n}(\varepsilon)
$$

SteP 3 : Estimate of $C_{n}(1, \varepsilon), C_{n}(2, \varepsilon)$ And $C_{n}(3, \varepsilon)$. Assume w. 1. o. g. that $\nu$ and $\lambda$ have the same sign. From Hölder's inequality, we deduce that with $p=\lambda / \nu, q^{-1}+p^{-1}=1$,

$$
0 \leq C_{n}(1, \varepsilon) \leq\left(\mathbf{E}\left(e^{\lambda \tau^{\varepsilon}}\right)\right)^{1 / p}\left(\mathbf{E}\left(\left|g\left(X_{\tau^{\varepsilon}}^{\varepsilon}\right)-u^{n}\left(\widehat{X}_{\tau^{\varepsilon}}^{\varepsilon}\right)\right|^{2 q}\right)\right)^{1 / q}
$$

Fix $n \in \mathbb{N}$. Thanks to the tightness of $\left(X_{s}^{\varepsilon}, \hat{X}_{s}^{\varepsilon}, \tilde{Y}_{s}^{\varepsilon, n}\right)$, we deduce from Proposition 3.5 that for each $n \geq 1$ fixed, $C_{n}(2, \varepsilon) \stackrel{\varepsilon \rightarrow 0}{\longrightarrow} 0$ and $C_{n}(3, \varepsilon) \stackrel{\varepsilon \rightarrow 0}{\longrightarrow} 0$.

Step 4 : Estimate of $C_{n}(4, \varepsilon)$. We shall take advantage of Proposition 3.2. For this end for any $\delta>0$ we consider a function $\varphi_{\delta} \in \mathcal{C}\left(\mathbb{R}^{d},[0,1]\right)$ satisfying

$$
\varphi_{\delta}(x)= \begin{cases}1, & x \in G \backslash G^{\delta}, \\ 0, & \text { in } G^{2 \delta}\end{cases}
$$


We have for every $\varepsilon>0$ and $n \geq 1$,

$$
\begin{aligned}
C_{n}(4, \varepsilon) & =2 \mathbf{E} \int_{0}^{\tau^{\varepsilon}} e^{\nu s} \widetilde{Y}_{s}^{\varepsilon, n} \delta_{1, n}(\varepsilon, s)\left(1-\varphi_{\delta}\left(\widehat{X}_{s}^{\varepsilon}\right)\right) d s+2 \mathbf{E} \int_{0}^{\tau^{\varepsilon}} e^{\nu s} \widetilde{Y}_{s}^{\varepsilon, n} \delta_{1, n}(\varepsilon, s) \varphi_{\delta}\left(\widehat{X}_{s}^{\varepsilon}\right) d s \\
& =C_{n}(4.1, \varepsilon, \delta)+C_{n}(4.2, \varepsilon, \delta) .
\end{aligned}
$$

For any $\delta>0$, all the arguments in the integral defining $C_{n}(4.1, \varepsilon, \delta)$ are bounded, uniformly w. r. t. $\varepsilon>0$. So using Proposition 3.2, we deduce that thanks to Lebesgue's theorem, uniformly w. r. t. $\varepsilon>0$,

$$
\mathbf{E} \int_{0}^{\tau^{\varepsilon}} e^{\nu s} \widetilde{Y}_{s}^{\varepsilon, n} \delta_{1, n}(\varepsilon, s)\left(1-\varphi_{\delta}\left(\widehat{X}_{s}^{\varepsilon}\right)\right) d s \rightarrow 0, \text { as } n \rightarrow \infty .
$$

Moreover we have

$$
C_{n}(4.2, \varepsilon, \delta) \leq C_{3.6} \mathbf{E} \int_{0}^{\tau^{\varepsilon}} e^{\nu s}\left|\delta_{1, n}(\varepsilon, s)\right| \varphi_{\delta}\left(\widehat{X}_{s}^{\varepsilon}\right) d s .
$$

Using Proposition 2.7, we deduce that for every $t \geq 0$,

$$
\mathbf{E} \int_{0}^{\tau^{\varepsilon}} e^{\nu s}\left|\delta_{1, n}(\varepsilon, s)\right| \varphi_{\delta}\left(\widehat{X}_{s}^{\varepsilon}\right) d s \stackrel{\varepsilon \rightarrow 0}{\longrightarrow} \mathbf{E} \int_{0}^{\tau} e^{\nu s}\left|F_{n}\left(X_{s}\right)\right| \varphi_{\delta}\left(X_{s}\right) d s
$$

with $F_{n}\left(X_{s}\right)=\bar{L} u^{n}\left(X_{s}\right)+\bar{f}\left(X_{s}, u^{n}\left(X_{s}\right), \partial_{x} u^{n}\left(X_{s}\right)\right), \quad n \geq 1$. Let us prove that

$$
\lim _{\delta \rightarrow 0} \sup _{n \geq 1} \mathbf{E} \int_{0}^{\tau} e^{\nu s}\left|F_{n}\left(X_{s}\right)\right| \varphi_{\delta}\left(X_{s}\right) d s=0 .
$$

To this end we consider two cases :

Case 1: $\lambda<0$. This implies $\nu<0$. Thanks to assumption (H1.6) and Krylov's estimate (see [10, Theorem 2.2]) there exists an integer $p>d$ and a constant $\widetilde{K}>0$ depending on $d, p, \nu$ and the diameter of the region $G$ such that

$$
\mathbf{E} \int_{0}^{\tau} e^{\nu s}\left|F_{n}\left(X_{s}\right)\right| \varphi_{\delta}\left(X_{s}\right) d s \leq \widetilde{K}\left(\int_{(0, \infty) \times G} e^{p \nu s}\left|F_{n}(x)\right|^{p} \varphi_{\delta}(x) d x d s\right)^{1 / p}
$$

Then Hölder's inequality implies

$$
\sup _{n \geq 1} \mathbf{E} \int_{0}^{\tau} e^{\nu s}\left|F_{n}\left(X_{s}\right)\right| \varphi_{\delta}\left(X_{s}\right) d s \leq \widetilde{K}\left(\int_{(0, \infty) \times G} e^{p \nu s} \varphi_{\delta}(x) d x d s\right)^{1 / 2 p}\left(\sup _{n \geq 1} \int_{(0, \infty) \times G} e^{p \nu s}\left|F_{n}(x)\right|^{2 p} d x d s\right)^{1 / 2 p} .
$$

Since $\nu<0$ and $F_{n}(\cdot)$ is bounded in $L^{2 p}(G)$ (thanks to Proposition 3.2 (iv)), the last term of the right hand side is finite. Moreover the sequence $\left(\varphi_{\delta}(x)\right)_{\delta>0}$ is decreasing to 0 at any point $x \in G$, as $\delta \downarrow 0$. As a consequence we have

$$
\lim _{\delta \rightarrow 0} \int_{(0, \infty) \times G} e^{p \nu s} \varphi_{\delta}(x) d x d s=0 .
$$


(3.13) follows in case 1.

Case 2: $\lambda>0$. Let $0 \vee \nu<\gamma<\lambda$. Using Hölder's inequality with $p=\lambda / \gamma>1$ and $q$ s.t. $p^{-1}+q^{-1}=1$, we have

$$
\begin{aligned}
\mathbf{E} \int_{0}^{\tau} e^{\nu s}\left|F_{n}\left(X_{s}\right)\right| \varphi_{\delta}\left(X_{s}\right) d s & =\mathbf{E} \int_{0}^{\tau} e^{\gamma s} \varphi_{\delta}\left(X_{s}\right) e^{(\nu-\gamma) s}\left|F_{n}\left(X_{s}\right)\right| d s \\
& \leq\left(\mathbf{E} \int_{0}^{\tau} e^{\lambda s} \varphi_{\delta}\left(X_{s}\right) d s\right)^{1 / p} \times\left(\mathbf{E} \int_{0}^{\tau} e^{q(\nu-\gamma) s}\left|F_{n}\left(X_{s}\right)\right|^{q} d s\right)^{1 / q}
\end{aligned}
$$

Using again Krylov's estimate, we deduce that (for some $m>d$ and $\widetilde{K}>0$ depending on the region $G, q, \nu-\gamma$ and $d$ )

$$
\sup _{n \geq 1} \mathbf{E} \int_{0}^{\tau} e^{q(\nu-\gamma) s}\left|F_{n}\left(X_{s}\right)\right|^{q} d s \leq \widetilde{K}\left(\sup _{n \geq 1} \int_{(0, \infty) \times G} e^{m q(\nu-\gamma) s}\left|F_{n}(x)\right|^{m q} d x d s\right)^{1 / m}<\infty
$$

thanks to Proposition 3.2 (iv) and $\nu-\gamma<0$. Moreover by uniform integrability, we have

$$
\lim _{\delta \rightarrow 0} \mathbf{E} \int_{0}^{\tau} e^{\lambda s} \varphi_{\delta}\left(X_{s}\right) d s=0
$$

(3.13) follows in case 2.

Step 5 : Proof of (ii). It follows from (3.13) that we can first choose $\delta(\eta)>0$ small enough, such that

$$
\sup _{n \geq 1} \lim _{\varepsilon \rightarrow 0} C_{n}(4.2, \varepsilon, \delta(\eta)) \leq \eta^{2} / 3 .
$$

From Proposition 3.2 (ii) and (3.12), we can next choose $n(\eta)$ large enough, such that (with $p=\lambda / \nu)$ both

$$
\begin{aligned}
& \sup _{x \in \partial G}\left|u^{n(\eta)}-g\right|(x) \leq\left(\sup _{\varepsilon>0, x \in \bar{G}} \mathbf{E}_{x}\left(e^{\lambda \tau^{\varepsilon}}\right)\right)^{-1 / 2 p} \sqrt{\eta^{2} / 3}, \\
& \sup _{\varepsilon>0} C_{n(\eta)}(4.1, \varepsilon, \delta(\eta)) \leq \eta^{2} / 3 .
\end{aligned}
$$

We now deduce from the above estimates, in particular (3.11), that

$$
\begin{gathered}
\limsup _{\varepsilon \rightarrow 0} C_{n(\eta)}(1, \varepsilon) \leq \eta^{2} / 3, \\
C_{n(\eta)}(2, \varepsilon)+C_{n(\eta)}(3, \varepsilon) \rightarrow 0, \quad \text { as } \varepsilon \rightarrow 0 .
\end{gathered}
$$

(ii) now follows from (3.10), (3.14), (3.15), (3.16) and (3.17). 


\section{Proofs of Propositions and Corollary 3.2 to 3.5}

\subsection{Proof of Proposition 3.2}

To begin with let us establish some preliminaries result. Since $g \in \mathcal{C}^{2}(\partial G)$ and $\partial G$ is of class $\mathcal{C}^{2}$ there exists a function $v \in W^{2, p}(G)$ such that $v(x)=g(x), x \in \partial G$.

Putting $\Psi(x, r, q)=-\bar{L} v(x)-\bar{f}\left(x, v(x)+r, \partial_{x} v+q\right),(x, r, q) \in G \times \mathbb{R} \times \mathbb{R}^{d}$, we have the following consequence of $[2$, Theorem 2].

Lemma 4.1. Assume that (H1) and (H2) are in force. Then the PDE

$$
\left\{\begin{array}{l}
\bar{L} u(x)+\Psi(x, u(x), \partial u(x))=0, \quad x \in G, \\
u(x)=0, \quad x \in \partial G .
\end{array}\right.
$$

admits at least one solution $u \in H_{0}^{1}(G)$.

As a consequence, we have

Proposition 4.2. The unique viscosity solution $u$ of equation (3.4) satisfies

$$
u \in W^{2, p}(G), \quad \text { for any } p \geq 1
$$

Proof. Let us consider $\bar{u}=u-v$. It suffices to prove that $\bar{u} \in W^{2, p}(G) \cap W_{0}^{1, p}(G), p \geq 1$. Let $\bar{v} \in H_{0}^{1}(G)$ be a solution of (4.1). It follows from $(H 2.2)$ that $\Psi(\cdot, \bar{v}(\cdot), \partial \bar{v}(\cdot)) \in L^{2}(G)$. Consequently, from $[1], \bar{v} \in W^{2,2}(G) \cap W_{0}^{1,2}(G)$. We then deduce that $\partial_{x} \bar{v} \in H^{1}(G) \hookrightarrow L^{p}(G)$ and $\bar{v} \in L^{p}(G)$ for all $p \geq 2$ if $d \leq 2$, for $p=2 d(d-2)^{-1}$ otherwise, by Sobolev embedding. From the linear growth of $\bar{f}$, we deduce that $\Psi(\cdot, \bar{v}(\cdot), \partial \bar{v}(\cdot)) \in L^{p}(G)$. This implies, again by [1],

$$
\bar{v} \in W^{2, p}(G) \quad \text { and } \quad \partial_{x} \bar{v} \in W^{1, p}(G) .
$$

Using again the Sobolev embedding, we deduce that $\bar{v}$ and $\partial_{x} \bar{v}$ belong to $L^{q}(G)$ for all $q$ if $d \leq 4$, for $q=2 d(d-4)^{-1}$ otherwise. Iterating this argument $\left\lceil\frac{d}{2}-1\right\rceil$ times, we deduce that $\bar{v} \in W^{2, p}(G)$ for all $p \geq 1$. Thus $\bar{v}$ is a viscosity solution of (4.1). Now uniqueness of the viscosity solution of the elliptic PDE (4.1) (see [15, Corollary 6.96]) implies $\bar{u}=\bar{v}$. The result follows since $v \in W^{2, p}(G)$ for all $p \geq 1$.

We are now in position to prove Proposition 3.2. To this purpose, we first extend the function $u$ as an element of $W^{2, p}\left(\mathbb{R}^{d}\right)$, which is possible given the regularity of $\partial G$ and $u$. Let $\rho: \mathbb{R}^{d} \rightarrow \mathbb{R}$ be a smooth mollifier with compact support, and define for $n \geq$ 1, $\rho_{n}(x)=n^{d} \rho(n x)$. We regularize $u$, the solution of (3.4), by convolution : $u^{n}$ defined as $u^{n}(x)=\left(u * \rho_{n}\right)(x)$. Thanks to Proposition $4.2, u \in \mathcal{C}^{1}(\bar{G})$. This implies that $\left(\partial_{x} u^{n}\right)_{n \geq 1}$ is uniformly bounded and

$$
\left(u^{n}, \partial_{x} u^{n}\right) \rightarrow\left(u, \partial_{x} u\right), \quad \text { as } n \rightarrow \infty \text {, uniformly in } \bar{G} \text {. }
$$


(i) and (ii) are established. Let us prove (iii). Since $\bar{L} u^{n}=(\bar{L} u) * \rho_{n}$, we have

$$
\begin{aligned}
\bar{L} u^{n}(x)+\bar{f}\left(x, u^{n}(x), \partial_{x} u^{n}(x)\right) & =\left[\bar{L} u+\bar{f}\left(\cdot, u(\cdot), \partial_{x}(\cdot)\right)\right] * \rho_{n}(x) \\
& +\bar{f}\left(x, u^{n}(x), \partial_{x} u^{n}(x)\right)-\left[\bar{f}\left(\cdot, u(\cdot), \partial_{x}(\cdot)\right)\right] * \rho_{n}(x) \\
& :=A^{n}(x)+B^{n}(x)
\end{aligned}
$$

For all $x \in G^{\delta}, A^{n}(x)=0$ if $n$ is large enough such that $\operatorname{Supp}\left(\rho_{n}\right) \subset B(0, \delta)$, the unit ball of radius $\delta$. Moreover since $\bar{f}$ is continuous with respect to its second and third arguments and (4.2), we have $B^{n}(x) \rightarrow 0$ as $n \rightarrow \infty$, uniformly w. r. t. $x$.

Finally (iv) follows from the fact that, since $u$ has been extended to an element of $W^{2, p}\left(\mathbb{R}^{d}\right), u^{n}$ is bounded in $W^{2, p}(G)$.

\subsection{Proof of Proposition 3.3.}

From (3.5), we deduce thanks to Itô's formula applied to the function $(t, y) \mapsto e^{\lambda t} y^{2}$

$$
\begin{aligned}
e^{\lambda t \wedge \tau^{\varepsilon}}\left|Y_{t \wedge \tau^{\varepsilon}}^{\varepsilon, x}\right|^{2}+ & \int_{t \wedge \tau^{\varepsilon}}^{\tau^{\varepsilon}} e^{\lambda r}\left|Z_{r}^{\varepsilon, x}\right|^{2} d r=e^{\lambda \tau^{\varepsilon}}\left|g\left(X_{\tau^{\varepsilon}}^{\varepsilon}\right)\right|^{2}-\int_{t \wedge \tau^{\varepsilon}}^{\tau^{\varepsilon}} \lambda e^{\lambda r}\left|Y_{r}^{\varepsilon, x}\right|^{2} d r \\
& +2 \int_{t \wedge \tau^{\varepsilon}}^{\tau^{\varepsilon}} e^{\lambda r} Y_{r}^{\varepsilon, x} f(\Theta(\varepsilon, r)) d r-2 \int_{t \wedge \tau^{\varepsilon}}^{\tau^{\varepsilon}} e^{\lambda r} Y_{r}^{\varepsilon, x} Z_{r}^{\varepsilon, x} d B_{r} .
\end{aligned}
$$

Let $0<\gamma<1$ and $\beta>0$ such that $\lambda=2 \mu+\frac{K^{2}}{\gamma}+\beta$. Using standard estimates, we have

$$
2 Y_{r}^{\varepsilon, x} f(\Theta(\varepsilon, r)) \leq\left(2 \mu+\frac{K^{2}}{\gamma}+\beta\right)\left|Y_{r}^{\varepsilon, x}\right|^{2}+\gamma\left|Z_{r}^{\varepsilon, x}\right|^{2}+\frac{1}{\beta}\left|f\left(X_{r}^{\varepsilon, x}, \bar{X}_{r}^{\varepsilon, x}, 0,0\right)\right|^{2}
$$

Hence we deduce from (4.3)

$$
\begin{aligned}
e^{\lambda t \wedge \tau^{\varepsilon}}\left|Y_{t \wedge \tau^{\varepsilon}}^{\varepsilon, x}\right|^{2}+(1-\gamma) \int_{t \wedge \tau^{\varepsilon}}^{\tau^{\varepsilon}} e^{\lambda r}\left|Z_{r}^{\varepsilon, x}\right|^{2} d r & \leq K^{2} e^{\lambda \tau^{\varepsilon}}+\frac{1}{\beta} \int_{t \wedge \tau^{\varepsilon}}^{\tau^{\varepsilon}} e^{\lambda r}\left|f\left(X_{r}^{\varepsilon, x}, \bar{X}_{r}^{\varepsilon, x}, 0,0\right)\right|^{2} d r \\
& -2 \int_{t \wedge \tau^{\varepsilon}}^{\tau^{\varepsilon}} e^{\lambda r} Y_{r}^{\varepsilon, x} Z_{r}^{\varepsilon, x} d B_{r}
\end{aligned}
$$

It follows from Burkholder-Davis-Gundy's inequality that there exists a constant $C_{3.3}>0$ such that

$$
\mathbf{E}\left[\sup _{0 \leq t \leq \tau^{\varepsilon}} e^{\lambda t}\left|Y_{t}^{\varepsilon}\right|^{2}+\int_{0}^{\tau^{\varepsilon}} e^{\lambda s}\left|Z_{s}^{\varepsilon}\right|^{2} d s\right] \leq C_{3.3} \mathbf{E}\left(K^{2} e^{\lambda \tau^{\varepsilon}}+\int_{0}^{\tau^{\varepsilon}} e^{\lambda r}\left|f\left(X_{r}^{\varepsilon, x}, \bar{X}_{r}^{\varepsilon, x}, 0,0\right)\right|^{2} d r\right)
$$

which is enough to get the desired result thanks to asumption (H2.2) and (3.2). 


\subsection{Proof of Corollary 3.4.}

Let $T>0$. Note that the process $M_{t}^{\varepsilon}=\int_{0}^{t \wedge \tau^{\varepsilon}} Z_{r}^{\varepsilon, x} d B_{r}, 0 \leq t \leq T$ satisfies $C V_{T}^{0}\left(M^{\varepsilon}\right)=0$. Using standard estimates and Doob's inequality, we have for any $\varepsilon>0$,

$$
\sup _{0 \leq t \leq T} \mathbf{E}\left|M_{t}^{\varepsilon}\right| \leq \frac{1}{2}\left(1+\mathbf{E}\left(\sup _{0 \leq t \leq T}\left|M_{t}^{\varepsilon}\right|^{2}\right)\right) \leq \frac{1}{2}\left(1+4 \sup _{\varepsilon>0} \mathbf{E}<M^{\varepsilon}>_{T}\right)
$$

Since for every $0 \leq s \leq T$, $e^{\lambda s}\left(1 \vee e^{-\lambda T}\right) \geq 1$, we deduce that

$$
\sup _{\varepsilon>0} \sup _{0 \leq t \leq T} \mathbf{E}\left|M_{t}^{\varepsilon}\right| \leq \frac{1}{2}\left(1+4 \sup _{\varepsilon} \mathbf{E}\left(\left(1 \vee e^{-\lambda T}\right) \int_{0}^{T \wedge \tau^{\varepsilon}} e^{\lambda s}\left|Z_{s}^{\varepsilon, x}\right|^{2} d s\right)<\infty .\right.
$$

Consequently, the sequence $\left\{M_{t}^{\varepsilon}, 0 \leq t \leq T\right\}$ is tight in $\mathcal{D}\left([0, T], \mathbb{R}^{d}\right)$.

Futhermore let $0=t_{0}<t_{1}<\ldots<t_{n}=T$ be a partition of $[0, T]$, thanks to the assumptions on $f$ and standard estimates, we have for all $\varepsilon>0$,

$$
\begin{aligned}
\mathbf{E} \sum_{i=0}^{n-1}\left|\mathbf{E}\left(Y_{t_{i+1}}^{\varepsilon}-Y_{t_{i}}^{\varepsilon} \mid \mathcal{F}_{t_{i}}\right)\right| & \leq \mathbf{E} \int_{0}^{T \wedge \tau^{\varepsilon}}\left|f\left(\bar{X}_{s}^{\varepsilon}, X_{s}^{\varepsilon}, Y_{s}^{\varepsilon}, Z_{s}^{\varepsilon}\right)\right| d s \\
& \leq K \mathbf{E}\left(1 \vee e^{-\lambda T}\right) \int_{0}^{T \wedge \tau^{\varepsilon}} e^{\lambda s}\left(1+\left|Y_{s}^{\varepsilon}\right|+\left|Z_{s}^{\varepsilon}\right|\right) d s \\
& \leq K T\left(1 \vee e^{-\lambda T}\right)\left(1+\mathbf{E} \sup _{0 \leq s \leq T \wedge \tau^{\varepsilon}} e^{\lambda s}\left|Y_{s}^{\varepsilon}\right|^{2}+\mathbf{E} \int_{0}^{T \wedge \tau^{\varepsilon}} e^{\lambda s}\left|Z_{s}^{\varepsilon}\right|^{2} d s\right)
\end{aligned}
$$

From this and Proposition 3.3, we deduce that

$$
\sup _{\varepsilon>0}\left(\sup _{0 \leq t \leq T \wedge \tau^{\varepsilon}} \mathbf{E}\left|Y_{t}^{\varepsilon}\right|+C V_{T}^{0}\left(Y^{\varepsilon} \mathbf{1}_{\left[0, \tau^{\varepsilon}\right]}\right)\right)<\infty
$$

which implies that the sequence $\left(Y_{s}^{\varepsilon}\right)_{\varepsilon>0}$ satisfies the Meyer-Zheng tightness criteria.

\subsection{Proof of Proposition 3.5.}

Let us define $\widetilde{\Psi}(x, y)=\Psi(x, y)-\bar{\Psi}(y)$. For every $T>0$, and $\delta>0$, we have for any $\nu<\lambda$,

$$
\mathbf{P}\left(\left|\int_{0}^{\tau^{\varepsilon}} e^{\nu r} \widetilde{\Psi}\left(\bar{X}_{r}^{\varepsilon}, V_{r}^{\varepsilon}\right) d r\right|>\delta\right) \leq \mathbf{P}\left(\tau^{\varepsilon}>T\right)+\mathbf{P}\left(\left|\int_{0}^{T \wedge \tau^{\varepsilon}} e^{\nu r} \widetilde{\Psi}\left(\bar{X}_{r}^{\varepsilon}, V_{r}^{\varepsilon}\right) d r\right|>\delta\right)
$$

Since $\tau^{\varepsilon} \Longrightarrow \tau$, the sequence $\left(\tau^{\varepsilon}, \varepsilon>0\right)$ is tight and we can choose $T$ large enough such that $\sup _{\varepsilon>0} \mathbf{P}\left(\tau^{\varepsilon}>T\right)$ is arbitrary small. Moreover the second term of the hand right side is equal to

$$
\mathbf{P}\left(\left|\int_{0}^{T} e^{\nu r} \widetilde{\Psi}\left(\bar{X}_{r}^{\varepsilon}, V_{r}^{\varepsilon}\right) \times 1_{\left\{0 \leq r \leq \tau^{\varepsilon}\right\}} d r\right|>\delta\right) .
$$


Thanks to the tightness of the process $1_{\left\{0 \leq r \leq \tau^{\varepsilon}\right\}}$ in $\mathcal{D}([0, \infty))$, [12, Lemma 4.2] implies that

$$
\mathbf{P}\left(\left|\int_{0}^{T} e^{\nu r} \widetilde{\Psi}\left(\bar{X}_{r}^{\varepsilon}, V_{r}^{\varepsilon}\right) \times 1_{\left\{0 \leq r \leq \tau^{\varepsilon}\right\}} d r\right|>\delta\right) \rightarrow 0, \text { as } \varepsilon \rightarrow 0 .
$$

It remains to prove that the collection of random variables $\left\{\int_{0}^{\tau^{\varepsilon}} e^{\nu r} \widetilde{\Psi}\left(\bar{X}_{r}^{\varepsilon}, V_{r}^{\varepsilon}\right) d r, \varepsilon>0\right\}$ is uniformly integrable. Since $|\widetilde{\Psi}(x, v)| \leq 2 M(1+|v|)$,

$$
\left|\int_{0}^{\tau^{\varepsilon}} e^{\nu r} \widetilde{\Psi}\left(\bar{X}_{r}^{\varepsilon}, V_{r}^{\varepsilon}\right) d r\right| \leq 2 M \int_{0}^{\tau^{\varepsilon}} e^{\nu r}\left(1+\left|V_{r}^{\varepsilon}\right|\right) d r
$$

Now we consider two cases :

Case 1: $\lambda>0$. We consider the case $\nu>0$ only, from which the result follows for $\nu \leq 0$. (4.5) implies

$$
\left|\int_{0}^{\tau^{\varepsilon}} e^{\nu r} \widetilde{\Psi}\left(\bar{X}_{r}^{\varepsilon}, V_{r}^{\varepsilon}\right) d r\right| \leq \frac{2 M}{\nu}\left(e^{\nu \tau^{\varepsilon}}+2 e^{\nu \tau^{\varepsilon} / 2} \sup _{0 \leq r \leq \tau^{\varepsilon}} e^{\nu r / 2}\left|V_{r}^{\varepsilon}\right|\right)
$$

The collection of random variables $\left\{\xi_{\varepsilon}:=e^{\nu \tau^{\varepsilon}}, \varepsilon>0\right\}$ is tight since $\sup _{\varepsilon>0} \mathbf{E}\left[\left|\xi_{\varepsilon}\right|^{p}\right]<\infty$ with $p=\lambda / \nu>1$, by (3.2). Now choose $2<p<2 \lambda / \nu$, and let $q$ be such that $q^{-1}+p^{-1}=1$. Then from Young's inequality,

$$
e^{\nu \tau^{\varepsilon} / 2} \sup _{0 \leq r \leq \tau^{\varepsilon}} e^{\nu r / 2}\left|V_{r}^{\varepsilon}\right| \leq \frac{1}{p} e^{p \nu \tau^{\varepsilon} / 2}+\frac{1}{q} \sup _{0 \leq r \leq \tau^{\varepsilon}} e^{q \nu r / 2}\left|V_{r}^{\varepsilon}\right|^{q} .
$$

Tightness of the last right hand side follows from $p \nu / 2<\lambda$ and $q \nu / 2<\lambda$, since $q<2$, (3.2) and (3.8).

Case 2: $\lambda<0$. Then $\nu<0$ as well. We have for any $\varepsilon>0$,

$$
\begin{aligned}
\int_{0}^{\tau^{\varepsilon}} e^{\nu r}\left(1+\left|V_{r}^{\varepsilon}\right|\right) d r & =\int_{0}^{\tau^{\varepsilon}} e^{(\nu-\lambda) r} e^{\lambda r}\left(1+\left|V_{r}^{\varepsilon}\right|\right) d r \\
& \leq \frac{1}{\lambda-\nu}\left(1+\sup _{0 \leq r \leq \tau^{\varepsilon}} e^{\lambda r}\left|V_{r}^{\varepsilon}\right|\right)
\end{aligned}
$$

Since $\lambda<0$, we have $\mathbf{E}\left[\left(\sup _{0 \leq r \leq \tau^{\varepsilon}} e^{\lambda r}\left|V_{r}^{\varepsilon}\right|\right)^{2}\right] \leq \mathbf{E}\left[\sup _{0 \leq r \leq \tau^{\varepsilon}} e^{\lambda r}\left|V_{r}^{\varepsilon}\right|^{2}\right]$. Hence using standard estimates and (3.8), we deduce that the last right hand side is tight.

Acknowledgements : The authors wish to thank François Hamel and Emmanuel Russ for valuable discussions concerning the proof of Proposition 3.2, and Alessio Porretta for pointing out to us the reference [2].

The work of the second author has been supported by LATP at Université de Provence, Marseille and AIRES-Sud, a program from the French Ministry of Foreign and European Affairs implemented by the Institut de Recherche pour le Développement (IRD-DSF). 


\section{References}

[1] S. Agmon, A. Douglis, L. Nirenberg : Estimates near the boundary for solutions of elliptic partial differential equations satisfying general bopundary conditions, Comm. Pure Appl. Math. XII, 623-727, 1959.

[2] L. Boccardo, F. Murat, J-P. Puel : $L^{\infty}$ estimate for some nonlinear elliptic partial differential equations and application to an existence result, SIAM, J. Math. Anal. 23, 326-333, 1992.

[3] P. Briand, Y. Hu : Stability of BSDEs with random terminal time and homogenization of semilinear elliptic PDEs, Journal of Functional Analysis 155, 455-494, 1998.

[4] F. Delarue : Auxiliary SDEs for homogenization of quasilinear PDEs with periodic coefficients, Annals Probab. 32, 2305-2361, 2004.

[5] S.N. Ethier, T.G. Kurtz : Markov Processes, Wiley Series in Probability and Mathematical Statistics. John Wiley \& Sons Inc., New York, 1986.

[6] M. Freidlin : The Dirichlet problem for an equation with periodic coefficients depending on a small parameter, Teor. Verojatnost. i Primenen. 9, 133-139, 1964.

[7] M. Freidlin : Markov processes and differential equations: asymptotic problems. Lectures in Mathematics ETH Zürich. Birkhäuser Verlag, Basel, 1996.

[8] M. Hairer, E. Pardoux : Homogenization of periodic linear degenerate PDEs, Journal of Functional Analysis 255, 2462-2487, 2008.

[9] A. Jakubowski : A non-Skorohod topology on the Skorohod space, Electronic Journal of Probability 2, 1-21, 1997.

[10] N. V. Krylov : Controlled diffusions processes, Applications of Mathematics 14, Springer, 1980.

[11] P.A. Meyer, W.A. Zheng : Tightness criteria for laws of semartingales, Ann. Inst. Henri Poincaré 20, 353-372, 1984.

[12] E. Pardoux : Homogenization of a linear and semilinear Second Order Parabolic PDEs with Periodic Coefficients: A Probabilistic Approach, Journal of Functional Analysis 167, 498-520, 1999.

[13] E. Pardoux : BSDEs, weak convergence and homogenization of semilinear PDEs, in Nonlinear Analysis, Differential Equations and Control, F.H. Clarke and R.J. Stern, eds. 503-549, Kluwer Acad. Pub., 1999.

[14] E. Pardoux : Backward stochastic differential equations and viscosity solutions of systems of semilinear parabolic and elliptic PDEs of second order, in Stochastic Analysis and Related Topics VI: The Geilo Workshop, 1996, L. Decreusefond, J. Gjerde, B. Oksendal, A.S. Üstünel eds., Birkhäuser, 79-127, 1998.

[15] E. Pardoux, A. Rascanu : Stochastic differential equations, Backward SDEs, Partial differential Equations, to appear. 
[16] E. Pardoux, A. Yu Veretennikov : On Poisson equation and diffusion approximation 3, Annals of Probab. 33, 1111-1133, 2005.

[17] A.B. Sow, R. Rhodes, E. Pardoux : Homogenization of periodic semilinear parabolic degenerate PDEs, Annales de l'Institut Henri Poincaré/Analyse non linéaire 26, 979998, 2009 .

[18] L. Tartar : H-measures, a New Approach for Studying Homogenization, Oscillations and Concentration Effects in Partial Differential Equations, Proc. Roy. Soc. Edin. 115A 193-230, 1990. 